\title{
APLICAÇÃO DE UM CORANTE TIAZOLILAZO COMO INDICADOR ÁCIDO-BASE E DETERMINAÇÃO DAS SUAS CONSTANTES DE IONIZAÇÃO ÁCIDA
}

\author{
Regina Terumi Yamaki*, Daniel Rodrigues Vieira, Cleber Galvão Novaes, Hygor Rodrigues de Oliveira e Valfredo \\ Azevedo Lemos \\ Departamento de Química e Exatas, Universidade Estadual do Sudoeste da Bahia, Av. José Moreira Sobrinho, s/n, 45206-191 \\ Jequié - BA, Brasil \\ Sandra Carvalho \\ Departamento de Química, Instituto de Ciências Exatas, Universidade Federal de Minas Gerais, 31270-901 Belo Horizonte - MG, \\ Brasil
}

Recebido em 25/9/08; aceito em 3/3/09; publicado na web em 17/9/09

\begin{abstract}
APPLICATION OF A THIAZOLYLAZO DYE AS AN ACID-BASE INDICATOR AND DETERMINATION OF ITS ACID IONIZATION CONSTANTS. The $p K a$ values of the 6-[2'-(6'-methyl-benzothiazolylazo)]-1,2-dihydroxy-3,5-benzenedisulfonic acid (Me-BDBD) have been determined at $25{ }^{\circ} \mathrm{C}$, in $0.10 \mathrm{~mol} \mathrm{~L}^{-1} \mathrm{NaCl}$ medium by spectrophotometric method. The SQUAD computer program was used to process experimental data in $\mathrm{pH}$ range $1.78-11.54$ and $290-720 \mathrm{~nm}$. The $\mathrm{pKa}$ values obtained were $4.60 \pm 0.04$ and $9.48 \pm 0.02$. The Me-BDBD reagent was applied as indicator in titration of acid-base. The results were compared with phenolphthalein and bromocresol green indicators. Statistical $t$ and $F$ tests indicated that there were no statistically significant differences between the results for indicators with good agreement.
\end{abstract}

Keywords: thiazolylazo; acidity constants; acid-base indicator.

\section{INTRODUÇÃO}

Corantes tiazolilazo são uma classe importante de derivados do 2-aminotiazol muito utilizados como reagentes analíticos. Esses compostos formam complexos coloridos com vários íons metálicos, sendo, por isso, muito utilizados na quantificação de metais por espectrofotometria, ${ }^{1}$ e como indicadores nas titulações complexométricas. ${ }^{2}$ Os corantes tiazolilazo também têm sido empregados em métodos analíticos baseados na extração em fase sólida, cromatografia líquida, extração líquido-líquido e extração no ponto-nuvem. ${ }^{3}$

No estado sólido cristalino, os corantes tiazolilazo apresentam coloração vermelha, violeta ou marrom, sendo pouco solúveis em água, mas a solubilidade pode ser aumentada pela adição de solventes orgânicos. ${ }^{4}$ Em meio ácido ou em soluções levemente ácidas, esses compostos formam complexos com íons metálicos na razão metal/ ligante 1:1 ou uma mistura de complexos 1:1 e 1:2 e em meio básico predomina o complexo 1:2. No entanto, o estudo do equilíbrio ácidobase envolvendo estes compostos é pouco investigado. ${ }^{5}$ Conhecer as constantes de acidez desses reagentes é importante para elucidar os mecanismos envolvidos em procedimentos analíticos. A potenciometria é um bom método para o estudo do equilíbrio ácido-base e de formação de complexos. Contudo, este método tem seu uso limitado quando se trata de reagentes pouco solúveis. A determinação de $\mathrm{pKa}$ de substâncias com baixa solubilidade pode ser feita usando o método espectrofotométrico, o qual permite o uso de soluções muito diluídas (concentração de $10^{-5} \mathrm{~mol} \mathrm{~L}^{-1}$ ).

Indicadores ácido-base utilizados nas titulações de neutralização são moléculas orgânicas cromóforas que absorvem na região do visível e apresentam mudanças de cor com a variação da atividade hidrogeniônica do meio, indicando o grau de acidez deste ambiente. Esta propriedade advém do fato de que essas substâncias apresentam grupos ácidos fracos e que têm seu sistema cromóforo fortemente influenciado pelo grupamento ácido/básico.

*e-mail: yamaki@uesb.br
O ácido 6-[2'-(6'-metilbenzotiazolilazo)]-1,2-diidroxi-3,5benzenodissulfônico (Me-BDBD) (Figura 1) é um corante tiazolilazo que tem sido recentemente aplicado na determinação de íons metálicos. Esse composto foi impregnado em espuma de poliuretano e utilizado em sistema em linha para pré-concentração de íons $\mathrm{Cu}$ (II). ${ }^{6}$ Entretanto, o estudo do equilíbrio ácido-base dessa substância não foi ainda realizado.<smiles>Cc1ccc2nc(/N=N/c3c([S+](=O)([O-])[O-])cc([S+](=O)[O-])c(O)c3O)sc2c1</smiles>

Figura 1. Estrutura do Me-BDBD $\left(\mathrm{H}_{2} L^{2-}\right)$

Neste trabalho, foram determinadas as constantes de ionização do ácido Me-BDBD por espectrofotometria. Investigou-se, também, a aplicação deste reagente como indicador em titulações ácido-base.

\section{PARTE EXPERIMENTAL}

\section{Reagentes e soluções}

Todos os reagentes empregados no trabalho foram de grau e pureza analítica e as soluções foram preparadas com água destilada e desionizada utilizando o desionizador Permution.

\section{Síntese do ácido Me-BDBD}

O ácido 6-[2'-(6'-metilbenzotiazolilazo)]-1,2-diidroxi-3,5-benzenodissulfônico foi sintetizado a partir da reação de diazotação do 6-metil-2-aminobenzotiazol, e o sal de diazônio formado foi acoplado com o ácido 1,2-diidroxi-3,5-benzenodissulfônico. ${ }^{6}$ 


\section{Determinação das constantes de ionização ácida do Me-BDBD}

A solução do ácido Me-BDBD foi preparada em etanol na concentração de $7,00 \times 10^{-4} \mathrm{~mol} \mathrm{~L}^{-1}$. Alíquotas desta solução foram diluídas com água desionizada, para volume final de 15,00 mL, obtendo-se soluções do ácido Me-BDBD nas concentrações de $1,365 \times 10^{-4}$ e 4,66 x 10-5 mol L ${ }^{-1}$, contendo 13 e $6,6 \%$ de etanol, respectivamente. Solução de $\mathrm{NaOH}$ de concentração conhecida foi utilizada como titulante. A força iônica e a temperatura foram mantidas constantes a $0,10 \mathrm{~mol} \mathrm{~L}^{-1} \mathrm{em}$ $\mathrm{NaCl}$ e $25^{\circ} \mathrm{C}$, respectivamente.

As medidas de $\mathrm{pH}$ foram realizadas utilizando o potenciômetro Digimed DM-20 equipado com eletrodo de vidro combinado com eletrodo de prata-cloreto de prata. O eletrodo foi calibrado com soluções tampão Merck pH 4,00 e 7,00. Medidas espectrofotométricas e de pH foram feitas após a adição de cada alíquota do titulante, utilizando o espectrofotômetro Varian Modelo Cary 50.

\section{Programa SQUAD}

A análise dos dados espectrofotométricos foi feita usando o programa SQUAD. ${ }^{7}$ Este programa exige uma proposta inicial para o modelo químico, no qual são considerados todos os possíveis equilíbrios envolvidos na formação das diversas espécies, sendo atribuídos valores estimados para as respectivas constantes de ionização. Esses valores estimados são refinados pelo método dos mínimos quadrados não linear, procurando minimizar a somatória dos quadrados das diferenças entre os valores de absorvância calculados e os medidos. Após o término do refinamento o programa permite uma avaliação do resultado através do cálculo de alguns parâmetros. O desvio padrão na absorvância deve ser da ordem de 0,01 . Desvios padrões nos valores das constantes devem ser da ordem de $1 \%$ do valor da constante. Os desvios padrões nos espectros individuais também são muito úteis, pois valores muito discrepantes sugerem problemas experimentais. O programa calcula as absortividades molares e as concentrações de cada espécie. Estes valores são usados para traçar gráficos de absortividade molar em função do comprimento de onda e de distribuição das espécies em função do $\mathrm{pH}$, os quais são úteis para verificar a qualidade do resultado.

Estudo do Me-BDBD como indicador nas titulações de ácidos fortes com bases fortes, ácidos fracos com bases fortes e bases fracas com ácidos fortes

O erro da titulação, nas titulações de ácidos fortes com bases fortes, utilizando o corante Me-BDBD como indicador ácido-base, foi determinado titulando-se alíquotas de $25,00 \mathrm{~mL}$ da solução de $\mathrm{HCl}$ na concentração igual a $0,0995 \mathrm{~mol} \mathrm{~L}^{-1}$, com solução de $\mathrm{NaOH}$ $0,1059 \mathrm{~mol} \mathrm{~L}^{-1}$, em presença deste indicador. Durante a titulação, foram feitas medidas de $\mathrm{pH}$ para cada alíquota da solução de $\mathrm{NaOH}$ adicionada.

Para avaliar a sensibilidade e a precisão deste novo reagente como indicador nas titulações de ácidos fortes com bases fortes e ácidos fracos com bases fortes, foram realizadas, também, titulações usando a fenolftaleína. Nas titulações de ácidos fortes com bases fortes foram utilizadas 10 gotas da solução dos indicadores Me-BDBD e de fenolftaleína em alíquotas de $25,00 \mathrm{~mL}$ de solução de $\mathrm{HCl}$. As soluções desses indicadores foram preparadas em $50 \%$ de etanol, na concentração igual a $1,572 \times 10^{-3} \mathrm{~mol} \mathrm{~L}^{-1}$. Alíquotas de $25,00 \mathrm{~mL}$ da solução de $\mathrm{HCl}$ foram tituladas com solução de $\mathrm{NaOH}$ na concentração igual a $0,08915 \mathrm{~mol} \mathrm{~L}^{-1}$. Foram realizadas quatro titulações para cada indicador. Para o estudo da utilização do Me-BDBD como indicador, nas titulações de ácidos fracos com bases fortes, a solu- ção de $\mathrm{HCl}$ foi substituída por alíquotas de $25,00 \mathrm{~mL}$ da solução de $\mathrm{H}_{3} \mathrm{CCOOH}$, que foram tituladas com solução de $\mathrm{NaOH}$ na concentração igual a $0,09600 \mathrm{~mol} \mathrm{~L}^{-1}$, na presença dos indicadores fenolftaleína e Me-BDBD. Foram realizadas seis titulações para cada indicador.

Nas titulações de bases fracas com ácidos fortes avaliou-se a sensibilidade e a precisão do reagente Me-BDBD como indicador titulando-se com solução de $\mathrm{HCl}$, soluções contendo cerca de $0,1800 \mathrm{~g}$ de $\mathrm{Na}_{2} \mathrm{CO}_{3}$ dissolvido em $25 \mathrm{~mL}$ de água desionizada e 10 gotas da solução de Me-BDBD 1,572 x $10^{-3} \mathrm{~mol} \mathrm{~L}^{-1}$. Foram realizadas, também, titulações das soluções de $\mathrm{Na}_{2} \mathrm{CO}_{3}$ com 10 gotas da solução do indicador verde de bromocresol na concentração de $7,43 \times 10^{-4} \mathrm{~mol} \mathrm{~L}^{-1}$. Durante as titulações, ao ocorrer a primeira mudança de cor aqueceu-se a solução para eliminar o $\mathrm{CO}_{2}$ eventualmente presente. Foram realizadas quatro titulações para cada indicador.

\section{RESULTADOS E DISCUSSÃO}

\section{Características espectrais do Me-BDBD}

A Figura 2 apresenta espectros de absorvância em função do comprimento de onda em vários valores de $\mathrm{pH}$, da solução do Me-BDBD na concentração de $1,365 \times 10^{-4} \mathrm{~mol} \mathrm{~L}^{-1}$, contendo $13 \%$ de etanol.

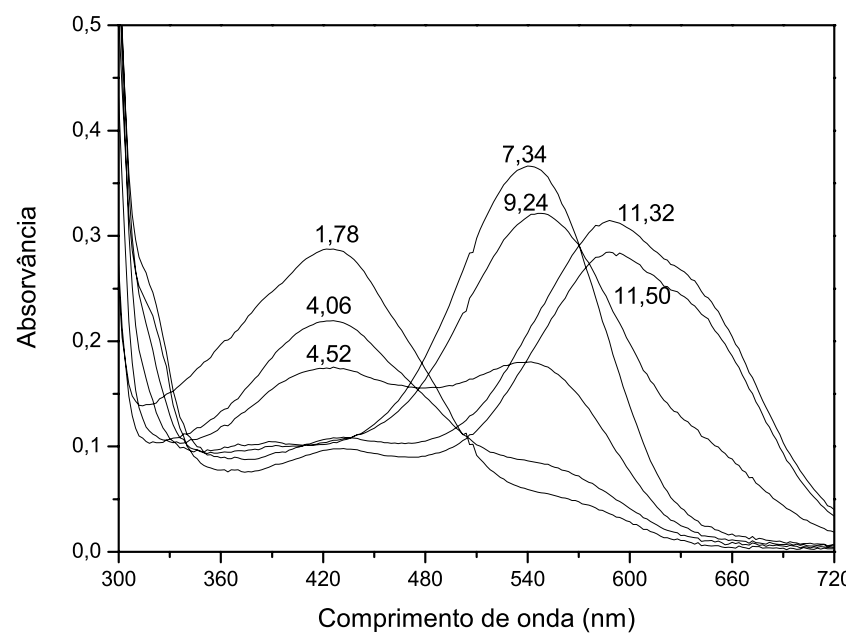

Figura 2. Espectros de absorção da solução do Me-BDBD na concentração de $1,365 \times 10^{-4} \mathrm{~mol} \mathrm{~L}^{-1}$ contendo $13 \%$ de etanol, em diferentes valores de $\mathrm{pH}$

Observa-se que o Me-BDBD apresenta máximos no espectro de absorção em 428, 542 e 586 nm em valores de pH 1,78; 7,34 e 11,32, respectivamente. Soluções de Me-BDBD de concentração 1,6 x 10 $0^{-3}$ mol L ${ }^{-1}$ apresentam coloração alaranjada ( $\left.\mathrm{pH} 1,8\right)$, rósea $(\mathrm{pH} 7,3)$ e violeta (pH 11,3) (Figura 3). A Figura 4 apresenta as estruturas do Me-BDBD em pH 1,78; 7,34 e 11,32.

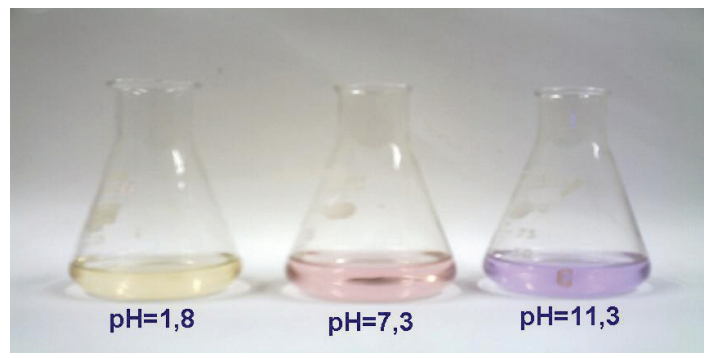

Figura 3. Variação na coloração das soluções de Me-BDBD na concentração igual a 1,6 $10^{-3} \mathrm{~mol} / \mathrm{L}$, de acordo com valores de pH de máxima absorção 


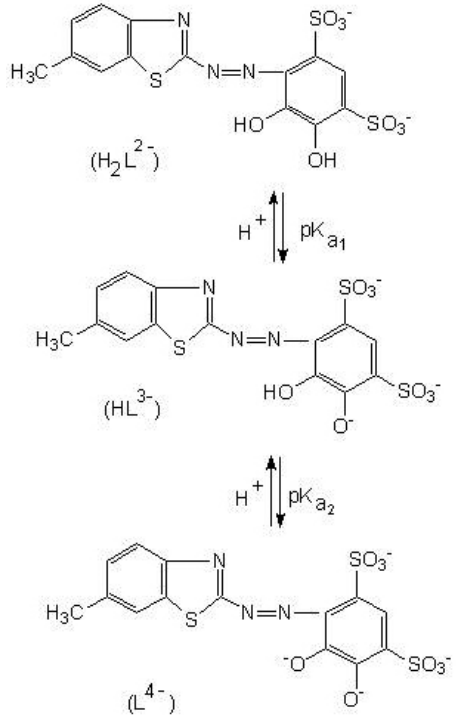

Figura 4. Estrutura das espécies do Me-BDBD $\mathrm{H}_{2} \mathrm{~L}^{2-}(\mathrm{pH}=1,78), H L^{3-}(\mathrm{pH}$ $=7,34) e L^{4-}(p H=11,32)$

\section{Tratamento dos dados}

Os dados espectrofotométricos tratados com o programa SQUAD estão na faixa de $\mathrm{pH}$ de 1,70 a 11,00 e comprimento de onda entre 290 a $720 \mathrm{~nm}$ com intervalo de $8 \mathrm{~nm}$. Para o ácido Me-BDBD foram estimados dois valores de constante de ionização ácida, atribuídos aos grupos $\mathrm{OH}$. Os resultados obtidos com o programa SQUAD estão apresentados na Tabela 1.

Tabela 1. Valores das constantes de ionização ácida do ácido MeBDBD obtidos a $25^{\circ} \mathrm{C}$, na força iônica $0,10 \mathrm{~mol} \mathrm{~L}^{-1} \mathrm{em} \mathrm{NaCl}$

\begin{tabular}{lcccc}
\hline Etanol (\%) & $p K_{a 1}$ & $p K_{a 2}$ & $\sigma_{\mathrm{A}}{ }^{*}$ & $\mathrm{U} * *$ \\
\hline 13 & $4,60 \pm 0,04$ & $9,48 \pm 0,02$ & $9,47 \times 10^{-3}$ & $7,73 \times 10^{-2}$ \\
6,6 & $4,55 \pm 0,03$ & $9,43 \pm 0,02$ & $3,26 \times 10^{-3}$ & $9,18 \times 10^{-3}$ \\
\hline
\end{tabular}

*desvio padrão da absorvância; **soma das diferenças entre valores calculados e medidas de absorvância

Observa-se que os valores das constantes de ionização ácida obtidos são próximos mesmo com a variação da quantidade de etanol. Os valores de $p K_{a l}$ e $p K_{a 2}$ são atribuídos ao grupo $\mathrm{OH}$ em posição meta e em posição orto, respectivamente (Figura 4). Esses valores estão de acordo com os valores encontrados na literatura para compostos similares. ${ }^{4}$ A Figura 5 apresenta os valores das absortividades molares em função do comprimento de onda, para as espécies do Me-BDBD, calculadas com o auxílio do programa SQUAD. Os valores das absortividades molares encontrados para as espécies $\mathrm{H}_{2} \mathrm{~L}^{2-}, \mathrm{HL}^{3-} \mathrm{e} \mathrm{L}^{4-} \mathrm{em}$ 428,536 e $596 \mathrm{~nm}$ foram iguais a 2.090, 3.130 e $2.990 \mathrm{~mol}^{-1} \mathrm{~L} \mathrm{~cm}^{-1}$, respectivamente.

\section{Indicador nas titulações de ácidos fortes com bases fortes, ácidos fracos com bases fortes e bases fracas com ácidos fortes}

Como indicador ácido/base, este reagente pode apresentar dois intervalos de viragem. Observam-se, na Figura 2, três bandas de absorção na região do visível, que surgem à medida que o $\mathrm{pH}$ varia. O primeiro intervalo ocorre no meio ácido em $\mathrm{pH}$ entre 3,60-5,60, com mudança de cor de alaranjado para rosa, e o segundo intervalo de viragem ocorre no meio básico em $\mathrm{pH}$ entre 8,48-10,48, com a variação da cor de rosa para violeta.

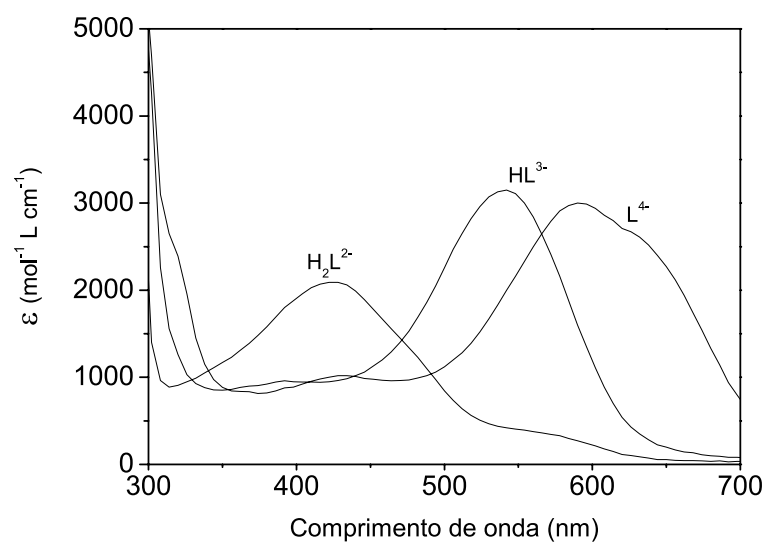

Figura 5. Absortividade molar das espécies do Me-BDBD em função do comprimento de onda

Para calcular o erro da titulação, foi considerado como volume teórico o volume de $\mathrm{NaOH}$ gasto no $\mathrm{pH}$ igual a 7,00 $\pm 0,01$. O intervalo de confiança obtido para o volume teórico, no nível de confiança de $95 \%$, foi igual a $23,49 \pm 0,084 \mathrm{~mL}$. Os valores obtidos para o intervalo de confiança de volume da primeira e da segunda viragens, foram iguais a 23,34 $\pm 0,070$ e 23,64 $\pm 0,045 \mathrm{~mL}$, respectivamente. Portanto, foi obtido o mesmo valor para o erro da titulação da primeira e da segunda viragens, que foi igual a $0,64 \%$.

Para testar a eficiência do Me-BDBD como indicador nas titulações de ácidos fortes com bases fortes e ácidos fracos com bases fortes, fez-se a comparação dos valores das concentrações das soluções de $\mathrm{HCl}$ e $\mathrm{H}_{3} \mathrm{CCOOH}$ obtidos nas titulações utilizando a fenolftaleína e o Me-BDBD como indicadores; a Tabela 2 apresenta esses valores.

Tabela 2. Valores das concentrações das soluções de $\mathrm{HCl} \mathrm{e} \mathrm{H}_{3} \mathrm{CCOOH}$ obtidos utilizando os indicadores fenolftaleína e Me-BDBD

\begin{tabular}{lcc}
\hline Ácido & $\begin{array}{c}\text { Fenolftaleína } \\
\text { Concentração }\left(\mathrm{mol} \mathrm{L}^{-1}\right)\end{array}$ & $\begin{array}{c}\text { Me-BDBD } \\
\text { Concentração }\left(\mathrm{mol} \mathrm{L}^{-1}\right)\end{array}$ \\
\hline $\mathrm{HCl}$ & $0,1012 \pm 4,3 \times 10^{-4}$ & $0,1009 \pm 2,9 \times 10^{-4} *$ \\
& & $0,1016 \pm 3,0 \times 10^{-4} * *$ \\
$\mathrm{H}_{3} \mathrm{CCOOH}$ & $0,1024 \pm 2,2 \times 10^{-4}$ & $0,1023 \pm 3,0 \times 10^{-4}$ \\
\hline
\end{tabular}

* primeira viragem, $* *$ segunda viragem

$\mathrm{O}$ teste $t$ indicou que os resultados das titulações do $\mathrm{HCl}$ e $\mathrm{H}_{3} \mathrm{CCOOH}$ utilizando o indicador fenolftaleína e o Me-BDBD, não apresentaram diferenças significativas para o nível de confiança de $95 \%$.

Os resultados das titulações do $\mathrm{Na}_{2} \mathrm{CO}_{3}$ com $\mathrm{HCl}$, utilizando o Me-BDBD e o verde de bromocresol como indicadores, estão apresentados na Tabela 3 . O teste $t$ indicou que esses resultados também não apresentaram diferenças significativas para o nível de confiança de $95 \%$.

A precisão dos resultados obtidos em todas as titulações utilizando o Me-BDBD como indicador foi também bastante satisfatória, sendo comprovada pelo teste $\mathrm{F}$ para o nível de confiança de $95 \%$.

Tabela 3. Valores da concentração da solução de $\mathrm{HCl}$ obtidos utilizando os indicadores verde de bromocresol e Me-BDBD

\begin{tabular}{lll}
\hline Ácido & $\begin{array}{l}\text { Verde de bromocresol } \\
\text { Concentração }(m o l ~ L-1)\end{array}$ & $\begin{array}{l}\text { Me-BDBD } \\
\text { Concentração }(m o l ~ L-1)\end{array}$ \\
\hline $\mathrm{HCl}$ & $0,1022 \pm 9,0 \times 10^{-4}$ & $0,1031 \pm 3,0 \times 10^{-4}$ \\
\hline
\end{tabular}




\section{CONCLUSÕES}

As constantes de ionização ácida do reagente Me-BDBD foram determinadas com sucesso, utilizando o programa SQUAD. Os resultados obtidos do estudo da aplicação do reagente Me-BDBD como indicador ácido-base, para titulações de ácidos fortes com bases fortes, ácidos fracos com bases fortes e bases fracas com ácidos fortes comprovam a sua eficiência e a viragem é de fácil detecção.

\section{AGRADECIMENTOS}

À FINEP, FAPESB ao CNPq pelo auxílio financeiro.

\section{REFERÊNCIAS}

1. Fan, X.; Zhu, C.; Microchem. J. 1998, 59, 284; Hnilicko, M.; Sommer, L.; Talanta 1969, 16, 83 .

2. Kai, F.; Izumi, H.; Anal. Lett. 1970, 3, 307; Chromy, V; Sommer, L.; Talanta 1967, 14, 393; Kai, F.; Anal. Chim. Acta 1969, 44, 129.

3. Lemos, V. A.; Santos, E. S.; Santos, M. S.; Yamaki, R. T.; Microchim. Acta 2007, 158, 189.

4. Hovind, H. R.; Analyst 1975, 100, 769.

5. Sanchez, M. J.; Santana, B.; Pont, M. L. P.; Gonzalez, V.; Montelongo, F. G.; Polyhedron 1988, 7, 495; Sanchez, M. J.; Santana, B.; Jimenez, F.; Montelongo, F. G.; Polyhedron 1990, 9, 501; Ghasemi, J.; Niazi, A.; Kubista, M.; Elbergali, A.; Anal. Chim. Acta 2002, 455, 335; Ghasemi, J.; Niazi, A.; Maeder, M.; J. Braz. Chem. Soc. 2007, 18, 267.

6. Lemos, V. A.; Vieira, D. R.; Novaes, C. G.; Rocha, M. E.; Santos, M. S.; Yamaki, R. T.; Microchim. Acta 2006, 153, 193.

7. Leggett, D. J.; Kelly, S. L.; Shiue, L. R.; Wu, Y.; Chang, D.; Kadish, K. M.; Talanta 1983, 30, 579. 